International Institute for Applied Systems Analysis • A-2361 Laxenburg • Austria

Tel: +432236807•Fax: +43223671313•E-mail: info@iiasa.ac.at•Web: www.iiasa.ac.at

INTERIM REPORT IR-98-074/ September

\title{
The Folk Theorems in the Framework of Evolution and Cooperation
}

Alexander Vasin (vasin@cs.msu.su)

\section{Approved by}

Arkadii Kryazhimskii(kryazhim@mi.ras.ru, kryazhim@iiasa.ac.at)

Principal Investigator, Dynamic Systems

Interim Reports on work of the International Institute for Applied Systems Analysis receive only limited review. Views or opinions expressed herein do not necessarily represent those of the Institute, its National Member Organizations, or other organizations supporting the work. 


\section{Contents}

1 Introduction $\quad 1$

2 The formal definitions $\quad 2$

3 The dominance solution supporting an outcome which dominates some $\begin{array}{ll}\text { Nash equilibrium } & 3\end{array}$

4 The theorem for a trembling-hand perturbation of the model 5

5 Discussion $\quad 6$ 


\begin{abstract}
Proceeding from the latest versions of the Folk Theorems, the present paper shows that "natural" evolution of behavior in repeated games in human populations is a very unstable process which may be easily manipulated by outside forces. Any feasible and individually rational payoff of the game may be converted in the globally stable outcome by arbitrary small perturbation of the payoff functions in the repeated game. We show that this result also holds for a trembling-hand perturbation of the game, and prove a new version of the Folk theorem for this case. This conclusion is in contrast to Axelrod (1984), Sigmund and Nowak (1992) and some other researches of evolution of behavior in the repeated Prisoner's dilemma. We discuss the reasons of the difference in the results.
\end{abstract}




\title{
About the Author
}

\author{
Alexander Vasin \\ Faculty of Computational Mathematics \\ and Cybernetics \\ Moscow State University \\ Moscow, 119899, Russia \\ phone/fax: 095-9392491
}

\section{Acknowledgment}

This paper was initiated by discussions at the workshop "Growth and Adaptation in Evolutionary Systems" at IIASA in October, 1997. I thank Arkadii Kryazhimskii and participants of the workshop for useful discussions. The paper was completed during my visit to the University of Alicante. I am grateful to the Department of the Fundamental Economic Researches for hospitality. The work was supported by the Ministry of Education and Culture, the Direccion General de Enseñanza Superior, Spain, and by the Russian Fund for Basic Researches, grant 96-01-0999. My special gratitude to Francisko Marhuenda for his help. 


\title{
The Folk Theorems in the Framework of Evolution and Cooperation
}

\author{
Alexander Vasin
}

\section{Introduction}

The question if repetition does lead to cooperation has been widely discussed in the gametheoretic literature since the known paper by Axelrod (1984). Up till now the answer is ambiguous. Computer simulations started by this work and continued by Sigmund, Novak $(1990,1992)$ confirm survival and superiority of the behavior strategies which lead to cooperation. This result was also supported by several theoretic researches (Fudenberg and Maskin (1990) and Binmore and Samuelson (1992)). However, the Folk theorems for repeated games (see Van Damme, 1987, for the survey of this field) show that every individually rational outcome of the stage game can be supported at some Nash equilibrium of the repeated game. With respect to the evolution of cooperation, this means that cooperation is not distinguished among many other equilibrium forms of behavior.

The possibility still remained that the cooperative outcome is the only stable one in some sense, or at least it has some robustic domain of attraction. In this context, the recent version of the Folk theorem for the dominance solutions ( Vasin, 1994, 1997) is of special interest. Besides other advantages, the concept of iterated strict dominance elimination (see Moulin, 1986) is very useful for investigation of the game dynamics. For a wide class of game dynamical systems it is known that frequencies of all eliminated strategies converge to 0 as time tends to infinity. This class includes, in particular, the Cournot tatonnement (Moulin, 1984), replicator dynamics (Vasin, 1989) and selection dynamics (Nachbar,1990).

Our result (Vasin 1997) establishes that the set of strict dominance solution payoffs of perturbed finitely repeated games converges to the set of individually rational convex combinations of payoffs in the stage game as the number of repetitions tends to infinity and the perturbation value tends to 0 . With respect to evolution of cooperation, this means the fail of the conjecture on the special status of cooperative behavior in game dynamics, at least for repeated games with complete information and unbounded rationality of players.

In a typical case, the construction of the dominance solution corresponding to desirable behavior in the repeated game is similar to the recursive constructions of the subgame perfect equilibrium in Benoit and Krishna (1985) and Fudenberg and Maskin (1986). At every stage each individual has to either realize the corresponding path or punish "the last disturber", i.e., the last player (with the smallest index) who deviated from the rule, if he has not been already sufficiently punished.

The most important innovation we introduced is a special end-game construction. It may be interpreted as the perturbation of the repeated game payoffs by some operating center (called the Manipulator below) who rewards or penalizes players depending on their behavior during the game. The presence of such center interested in the outcome of the game is typical for social interactions. For instance, recall about the prosecutor in the original version of the Prisoner's dilemma. He is obviously uninterested in "cooperative" 
behavior of the "players". The whole concept of cooperative behavior is doubtful in such situations involving several persons with different interests and asymmetric positions.

Players' behavior corresponding to subgame perfect equilibria in Benoit and Krishna (1985) and Fudenberg and Maskin (1986) (as well as the dominance solution in our paper, 1997 ) is rather sophisticated. The present paper aims to provide a very simple construction of the dominance solution for every outcome where payoffs to all players are not less than their payoffs at some Nash equilibrium of the stage game. After any deviation, players just switch to playing the Nash equilibrium till the end of the repeated game. The "last disturber" looses his/her award. Thus, the idea of the solution is close to Radner (1980) who considered the Nash equilibria of a similar perturbation of the repeated oligopoly game. This case covers all individually rational outcomes in the Prisoner's dilemma and in the Coordination game with one efficient equilibrium. Then we generalize this result for a "trembling- hand" perturbation of a stage game according to Selten, 1975, that is, for the case where players mistake in their actions with a small probability. In conclusion we give a brief survey of results which confirm the convergence of evolutionary dynamics to the cooperative behavior strategies and discuss the reason of the difference with our results.

\section{The formal definitions}

Let $\Gamma$ be a normal form game with the set of players $I=\{1, \ldots, n\}$, the sets of strategies $X_{1}, \ldots, X_{n}$ and the payoff functions $f_{1}(x), \ldots, f_{n}(x), x \in \underset{i}{\bigotimes} X_{i}$. Let $\left(x \| y_{i}\right)$ denote the result of substitution of $y_{i}$ for $x_{i}$ in the strategy combination $x=\left(x_{i}, i \in I\right)$.

Strategy $x_{i}$ weakly dominates strategy $y_{i}$ on the set $\bar{X} \subseteq X$ if there exists $\epsilon \geq 0$ such that for any $z \in \bar{X}$

$$
f_{i}\left(z \| x_{i}\right) \geq f_{i}\left(z \| y_{i}\right)+\epsilon
$$

In contrast to the standard definition, we do not require the strict inequality for at least one $z \in \bar{X}$.

Strategy combination $\bar{x}$ is a weak dominance solution if it may be obtained by means of the weak dominance elimination procedure (see Moulin, 1981), that is to say, if there exists a sequence of sets $X=X^{1} \supset X^{2} \supset \ldots \supset X^{k}=\{\bar{x}\}$, where, for every $l=1, \ldots, k-1$, $X^{l}=\bigotimes_{i} X_{i}^{l}$, for any $x_{i} \in X_{i}^{l} \backslash X_{i}^{l+1}$ there exists $y_{i} \in X_{i}^{l+1}$ that weakly dominates $x_{i}$ on $X^{l} ; x_{i}$ strictly dominates $y_{i}$ on $\bar{X}$ if (1) holds for $\epsilon>0 ; \bar{x}$ is a strict dominance solution, if it can be obtained by means of successive elimination of strictly dominated strategies.

Consider a $T$-fold repetition $\Gamma_{T}$ of a normal form game $\Gamma$. In order to avoid confusion between strategies in the $\Gamma_{T}$ and strategies in the initial game, the latter will be referred to as actions. At any time $t$ every player knows the actions of all participants at previous periods. Let $x^{t} \in X$ be the action combination at time $t, h^{t}=\left(x^{0}, \ldots, x^{t-1}\right)$ - a history at this time, $X^{t}$ - the $t$-fold Cartesian product of $X, H^{T}=\bigcup_{t=0}^{T-1} X^{t}$ - the set of all histories to time $T\left(X^{0} \stackrel{\text { def }}{=}\{0\}\right)$. A strategy of player $i$ is a mapping $m_{i}: H^{T-1} \rightarrow X_{i}$. This mapping determines the choice of the action for every time $t$ and any history $h^{t}$. Each strategy combination $m=\left(m_{i}, i \in I\right)$ induces the path of $\Gamma_{T} h(m)=\left\{x^{t}(m)\right\}$, where $x^{0}(m)=0$, $x^{1}(m)=m(0), x^{t}(m)=m\left(x^{0}(m), \ldots, x^{t-1}(m)\right), t \geq 1$. The payoff function of player $i$ is given by 


$$
F_{i, T}(m)=F_{i, T}(h(m))=\sum_{t=0}^{T-1} f_{i}\left(x^{t}(m)\right) / T .
$$

For any game $\Gamma$ with sets of strategies $Y_{i}$ and payoff functions $u_{i}(y), i=1, \ldots, n$, and for any $\epsilon>0$, let $A(\Gamma, \epsilon)$ denote the set of games with the same sets of players and strategies and payoff functions $u_{i}^{\prime}(y)$ which differ from $u_{i}(y)$ less than by $\epsilon$. Since repeated game $\Gamma_{T}$ is a particular case of a normal form game, we may consider a set $A\left(\Gamma_{T}, \epsilon\right)$ of its perturbations. Let us stress that any perturbed repeated game $\hat{\Gamma} \in A(\tilde{\Gamma}, \epsilon)$ is obtained from $\tilde{\Gamma}$ by variation of its payoff functions. The set $A\left(\Gamma_{T}, \epsilon\right)$ is wider than the set of repeated games corresponding to perturbed stage games $\Gamma^{\prime} \in A(\Gamma, \epsilon)$.

Our result (1997) describes the limit set of the dominance solution payoffs of games $\hat{\Gamma} \in A\left(\Gamma_{T}, \epsilon\right)$ as $T$ tends to infinity and $\epsilon$ tends to 0 . Let $v_{i}$ be the minmax payoff of player $i$ :

$$
v_{i}=\min _{x} \max _{x_{i}} f_{i}\left(x \| x_{i}\right)
$$

Let $W=\{f(x), x \in X\}$ be the set of payoff vectors for pure strategies, $C o W$ denote the convex hull of $W$ and $\Phi=\{w \in C o W \mid w \geq v\}$ be the set of individually rational convex combinations of payoff vectors in the stage game $\Gamma ; M=\max _{x, z \in X}|f(x)-f(z)|$.

Theorem 1. (Vasin, 1997) The set of strict dominance solution payoff vectors of games $\hat{\Gamma} \in A\left(\Gamma_{T}, \epsilon\right)$ converges (in the sense of Hausdorff distance) to the set $\Phi$ as $T$ tends to $\infty$ and $\epsilon$ tends to 0 .

The construction of the dominance solution and the payoff perturbation are rather sophisticated in a general case. The next section studies the problem for a stage game $\Gamma$ with a Nash equilibrium $\bar{x}$.

\section{The dominance solution supporting an outcome which dominates some Nash equilibrium}

For any sequence $z_{1}, \ldots, z_{r}$ of action profiles such that $\sum_{k} f\left(z_{k}\right) / k \geq f(\bar{x})$, we describe a simple payoff perturbation and a strategy profile $m^{*}$ which supports repetition of this sequence at the dominance solution of the repeated game with the perturbed payoff functions.

In order to explain the construction of the dominance solution corresponding to a sequence of action profiles, let us consider a version of the coordination game with the set of actions $\{1,2,3\}$ and the payoff matrix

$\begin{array}{cccc} & 1 & 2 & 3 \\ 1 & (5,5,0) & (1,0,0) & (0,3,5) \\ 2 & (0,1,0) & (1,1,2) & (0,0,0) \\ 3 & (3,0,5) & (0,0,0) & (0,0,0)\end{array}$

where the third component shows the gain of the Manipulator who does not act but is interested in his payoff. More precisely, we assume that he would like to maximize his average payoff in the $T$-fold repetition. Thus, action profiles $(1,1)$ and $(2,2)$ are resp. the "cooperative" and the "bad" Nash equilibrium for the active players, and alternation between $(1,3)$ and $(3,1)$ is optimal for the Manipulator among sequences of action profiles which are individually rational for players 1,2 . 
If the Manipulator does not interfere then the natural outcome of the game is repetition of the cooperative equilibrium. Our purpose is to describe a perturbation of the payoff function in $\Gamma_{T}$ which supports alternation between $(1,3)$ and $(3,1)$ at the dominance solution of the perturbed repeated game. The corresponding strategy of each player is to repeat his/her actions in this sequence till the first deviation and to play action 2 any time after the deviation if it happens. The payoff function perturbation may be interpreted as "awards" which the Manipulator pays to the players for "good behavior" in this game. The value of the "award" is $6 / T$. If nobody deviates from alternating between $(1,3)$ and $(3,1)$ then the both players get their awards. Otherwise the player who is the last to deviate from the specified strategies is penalized. If the last deviation involved both players simultaneously then only player 1 does not get the award.

The weak dominance elimination may be realized as follows. At first we can exclude any strategy such that the first player deviates at time $T-1$ for some prehistory. If player 1 is the "last disturber" according to prehistory and player 2 uses action 2 at this time then this deviation is unprofitable. Otherwise player 1 gains at most $5 / T$ by deviation but loses $6 / T$ because he/she either becomes the last disturber or misses the chance to pass this label to player 2. At the next stage every strategy which permits deviation by player 2 at time $T-2$ is eliminated in a similar way. Now we can continue this reasoning for the time $T-2, T-3$, etc. After $2 T$ stages of eliminations, we obtain the desirable solution.

Thus, by spending $12 / T$ for awards, the Manipulator increases his average gain from 0 to 5 in this example.

Now, consider a general case where desirable behavior corresponds to repetition of $z_{1}, \ldots, z_{r}$. Then the strategy profile is as follows. The players repeat this sequence until at least one of them deviates from this choice. Then they switch to playing $\bar{x}$ at every repetition till the end of the game. In order to define the payoff perturbation, consider the last disturber, that is, the last player (with a minimal index) not to confirm to the specified behavior rule. This player is penalized with the fine $r M$. More formally,

$$
m_{i}^{*}\left(h^{t}\right)=z(\{t / m\}+1) \text { if } x^{s}=z(\{s / m\}+1) \text { for anys }<t, \text { otherwisem } m_{i}^{*}\left(h^{t}\right)=\bar{x}_{i} .
$$

For any path $h^{T}$, let

$$
s\left(h^{t}\right)=\max \left\{s<t \text { s.t. } x^{s} \neq m^{*}\left(h^{s}\right)\right\}
$$

denote the last time of deviation before $t$, and

$$
i\left(h^{t}\right)=\min \left\{i \text { s.t. } x_{i}^{s\left(h^{t}\right)} \neq m_{i}^{*}\left(h^{s\left(h^{t}\right)}\right)\right\}
$$

denote the last disturber to time $t$. The fine for deviation is

$$
\varphi_{i}\left(h^{T}\right)=r M / T \text { for } i=i\left(h^{T}\right)
$$

and is 0 for any other $i$. Finally, the perturbed payoff function is

$$
\hat{F}_{i}(m)=F_{i, T}(m)-\varphi_{i}(h(m)), i=1, \ldots, n .
$$

Proposition 1. Strategy profile $m^{*}$ defined according to (2) is a weak dominance solution of game $\hat{\Gamma}$ with payoff functions (3).

Proof actually repeats arguing for the example above. Consider the player 1 and any strategy $m_{1}$ such that $m_{1}\left(h^{T-1}\right) \neq m_{1}^{*}\left(h^{T-1}\right)$ for some $h^{T-1}$. Let us show that strategy $\bar{m}^{1}$, s.t. $\bar{m}_{1}\left(h^{t}\right)=m_{1}\left(h^{t}\right)$ for any $t<T-1$ and $\bar{m}_{1}\left(h^{T-1}\right)=m_{1}^{*}\left(h^{T-1}\right)$ for any $h^{T-1}$, weakly dominates $m_{1}$. Consider any strategy profile $m$ including $m_{1}$. If $h^{T-1}(m)=h^{T-1}\left(m^{*}\right)$ then deviating from $m_{1}^{*}\left(h^{T-1}(m)\right)$ is unprofitable since it makes player 1 the last disturber, 
and he has to pay the fine $r M / T$ while by deviating he gains at most $M / T$. Otherwise consider the last disturber to the time $T-1$. If $i(m, T-1) \neq 1$ or $x_{j}\left(h^{T-1}(m)\right) \neq \bar{x}_{j}$ for some $j \neq 1$ then by deviating player 1 is missing the chance to pass the label of the last disturber to another player, and we can argue as in the previous case. Otherwise deviating brings nothing since $\bar{x}$ is a Nash equilibrium. We can continue this reasoning by induction for $i=2,3, \ldots, n$ and then for $t=T-2, \ldots, 0$. The only correction is that by deviating at time $T-k$ a player can gain at $\operatorname{most} \min \{k, r\} M$.

\section{The theorem for a trembling-hand perturbation of the model}

Random mistakes introduce some difficulties in the proposed scheme of behavior regulation. Consider a game $G_{T, d}$ which is a trembling-hand perturbation of the repeated game $\Gamma_{T}$ according to Selten,1975. In this game, for any prehistory $h^{t}$ and strategy $m_{i}$, the action of player $i$ in period $t$ is $m_{i}\left(h^{t}\right)$ with probability $1-d$ and any other action with probability $d /\left(\left|X^{i}\right|-1\right)$. Every strategy profile $m$ determines a probability distribution $P_{d}\left(h^{T} \mid m\right)$ over the set of paths $h^{T}$. Let each player be interested in his/her expected payoff

$$
g_{i}(m)=\sum_{h^{T}} P_{d}\left(h^{T} \mid m\right) F_{i}\left(h^{T}\right)
$$

Assume that the desirable behavior of the players corresponds to action profile $x^{*}$ such that $f\left(x^{*}\right) \geq f(\bar{x})$ for some Nash equilibrium $\bar{x}$. Consider the strategy profile $m^{*}$ corresponding to $x^{*}$ according to the section 3 . Let us define the perturbed payoff functions as follows. For every path $h^{T}$, we can compute the last disturber to the time $T$ as above. Let him pay the fine $\bar{\varphi}$ while other players pay nothing. Formally, the value of the fine is given by the following function:

$$
\varphi_{i}\left(h^{T}\right)=\bar{\varphi} \text { if } i=i\left(h^{T}\right) \text { otherwise } \varphi_{i}\left(h^{T}\right)=0 .
$$

Consider a game $\hat{G}^{T}$ with payoff functions

$$
\hat{g}_{i}(m)=\sum_{h^{T}} p\left(h^{T} \mid m\right)\left(F_{i}\left(h^{T}\right)-\varphi_{i}\left(h^{T}\right)\right)
$$

Proposition 2. Let the value of the fine $\bar{\varphi}>M /\left(T a^{T}\right)$, where $a={ }^{d e f}(1-d)^{n}$. Then $m^{*}$ is a strict dominance solution of the game $\hat{G}^{T}$ if $1-d>d /\left|X_{i}\right|, i=1, \ldots, n$.

Proof. Assume that for every $i=1, \ldots, n$ we have already eliminated all strategies $m_{i}$ such that $m_{i}\left(h^{\tau}\right) \neq m_{i}^{*}\left(h^{\tau}\right)$ for some $\tau>t, h^{\tau}$. Consider any $h^{t}$ and $m_{1}$ such that $m_{1}\left(h^{t}\right)=z_{1} \neq m_{1}^{*}\left(h^{t}\right)$. Let us show that $\bar{m}_{1}$, such that $\bar{m}_{1}\left(h^{t}\right)=m_{1}^{*}\left(h^{t}\right), \bar{m}_{1}\left(\hat{h}^{\tau}\right)=$ $m_{1}\left(\hat{h}^{\tau}\right)$ for any other $\tau, h$, strictly dominates $m_{1}$. First consider the case where 1 is the last disturber after $h^{t}$. Then, according to $m^{*}$, the players have to play $\bar{x}$ since $t$ till the end of the game. Let $\hat{g}_{i}\left(h^{t}, m\right)$ denote the expected gain of player $i$ in $\hat{G}$ under history $h^{t}$ and profile $m$. Then, for any $d>0$,

$$
\begin{array}{r}
\hat{g}_{1}\left(h^{t}, m \| \bar{m}_{1}\right)-\hat{g}_{1}\left(h^{t}, m\right)>\min _{x}\left((1-d)^{2}-\left(d /\left|X_{i}\right|\right)^{2}\right)\left(\hat{g}_{1}\left(h^{t}, x \| \bar{x}_{1}, m^{*}\right)-\right. \\
\hat{g}\left(h_{1}^{t}, x \| z_{1}, m^{*}\right) .
\end{array}
$$

Consider the last difference. If $x=\bar{x}$ then 1 gains nothing by deviating since $\bar{x}$ is a Nash equilibrium, and behavior since time $t+1$ does not depend on $x_{1}^{t}$. Otherwise, by 
deviating from $\bar{x}_{1}$, player 1 gains at most $M$ but increases the probability to become the last disturber at the time $T$ and to pay the fine $\bar{\varphi}$ in $a^{(T-t-1)}$. Thus, $\bar{m}_{1} \succ m_{1}$.

The same reasoning works if some other player is the last disturber at time $t$. If nobody is then the only difference is that the expected total gain in repetitions since $t+1$ decreases by deviation since the players will have to switch from $x^{*}$ to $\bar{x}$. We can continue this arguing for players $2, \ldots, n$ and thus complete the proof.

Proposition 3. The mean time of playing $x^{*}$ in the game $\hat{G}$ under the strategy profile $m^{*}$ is

$$
\tau(T)=\left(1-a^{T}\right) /(1-a)-T a^{T-1}(1-a)
$$

Proof. The probability of playing $x^{*} t$ times,$t<T$, is $a^{t}(1-a)$, and for $T$ this probability is $a^{T}$. Thus, the mean time is $(1-a) \sum_{1}^{T-1} \tau a^{\tau}+T a^{T}$ which coincides with (5).

Proceeding from the propositions 2,3 , the profile $m^{*}$ does not ensure the desirable behavior for $T>1 / d$. Let $T=k / d$ for some $k>1$. Then, for $d$ small enough, $a \approx 1-n d$, $a^{T} \approx \exp (-n k)$, the mean share of the time of playing $x^{*}$ is $\tau(T) / T \approx\left(1-e^{-n k}\right) / n k-$ $n(1-n d) e^{-n k}$ and tends to 0 as $k$ tends to infinity, while the necessary amount of the fine is about $d M \exp (k n) / k$ and tends to infinity.

One possibility to improve the strategy is partitioning of the time of the game into $s$ intervals of the same length $\bar{T}=k / d$ and playing the profile $m^{*}$ independently in each interval. Let $m^{* *}$ denote this strategy combination. The corresponding payoff perturbation assumes that, after each interval, the players pay the fine (4) according to their behavior within this interval. Let $\varphi^{* *}\left(h^{T}\right)$ denote the corresponding perturbation of the total payoff. Then the maximal value of the perturbation is about $d M \exp (k n) / k$ for any small $d$ and $k \sim O\left(1 / d^{1 / s}\right), s \in Z$. Under $m^{* *}$, the mean time of playing $x^{*}$ related to $T$ is the same as in (5). Thus, for any game $\Gamma$ with a Nash equilibrium $\bar{x}$, we obtain the following result.

Theorem 2. For any $\varepsilon>0$, there exist $d \dot{d}>0$ and $T$ such that for any $d<\dot{d}$ and $T>\dot{T}$, every action profile $x^{*}$ such that $f_{i}\left(x^{*}\right) \geq f_{i}(\bar{x}), i=1, \ldots, n$, may be supported at the strict dominance solution $m$ of game $\hat{G} \in A\left(G_{T}, \varepsilon\right)$ such that the mean share of the time of playing $x^{*}$ under $m$ exceeds $1-\varepsilon$.

Proof. Consider the game $\hat{G}_{T}$ with the payoff perturbation $\phi^{* *}$. For $d$ small enough, the strategy profile $m^{* *}$ is a strict dominance solution if the fine exceeds $d M \exp (k n) / k$. The mean share of the time of playing $x^{*}$ is given by (4). In order to make this share more than $1-\varepsilon$, let us set $k$ such that $1-e^{-n k}>n k(1-\varepsilon)$. Since $e^{-n k}<1-n k+n k^{2} / 2$ it suffices to set $k=2 \varepsilon / n$. Now, in order to make the total payoff perturbation less then $\varepsilon$, choose $d$ such that $n d M e^{2 \varepsilon} / 2 \varepsilon<\varepsilon$. It suffices to take $\bar{d}=\varepsilon^{2} / n M$. Finally, $T$ should be large enough to divide it into equal intervals of length $k / d=4 M / \varepsilon$.

Corollary. For any game $\Gamma$ with Nash equilibrium $\bar{x}$ such that $v_{i}=f_{i}(\bar{x}), i=1, \ldots, n$, consider a trembling-hand perturbation $G_{T}$ of $T$-fold repetition of $\Gamma$. The set of the strict dominance solution payoffs in games $\hat{G}_{T} \in A\left(G_{T}, \varepsilon\right)$ converges to the set $\Phi$ of individually rational convex combination of payoffs in $\Gamma$ as $T$ tends to infinity and $\varepsilon$ tends to 0 .

Proof. The only difference with the proof of the theorem is that, instead of playing $x^{*}$ in every repetition, the desirable behavior in this case is a repeated sequence of action profiles $z 1, \ldots, z r$ such that $\sum_{k} f(z k) / r$ approximates a given $w \in \Phi$. The minor modifications include $r$-fold increasing of the fine. Thus, we have proved the Folk theorem for the dominance solutions of games in the specified class.

\section{Discussion}

One important direction in studying of evolution of cooperation is computer simulation of behavior dynamics in the iterated Prisoner's dilemma. Two players engaged in the 
Prisoner's dilemma have to choose between cooperation $(C)$ and defection $(D)$. In any given round, each player receives $R$ if both cooperate and payoff $P<R$ if both defect; but a defector exploiting a cooperator gets $T$ points, while the cooperator receives $S$ (with $T>R>P>S$ and $2 R>T+S$ ). Thus in a single round it is always best to defect, but cooperation may be rewarded in an iterated Prisoner's dilemma. Axelrod's Computer tournaments (see Axelrod, 1984) have shown that the known "tit for tat" strategy which supports cooperation wins the competition with other deterministic strategies where the decision to cooperate or defect in each round depends on the outcome of the three previous rounds. Nowak and Sigmund(1990) have considered the case where the decision depends only on the previous round, but is stochastic and not deterministic. Each strategy consisted of two parameters, $p^{C}$ and $p^{D}$. These give the probability of cooperating after a $C$ (cooperate) or $D$ (defect) by the other player. In these simulations Generous-TFT $\left(p^{C}=1\right.$ and $p^{D}=1 / 3$ given the usual payoffs) appeared to be a stable end state, as almost any starting condition converged to it provided the run was long enough.

Later Nowak and Sigmund (1992) extended this treatment to "memory 2" strategies, in which players base their decision on the other player's action as well as on their own previous action. They found a different strategy dominating the long-term behavior. They called this strategy "Pavlov" or "win-stay, lose-shift" (WSLS) because if it receives a good payoff (either $T$ or $R$ ) it repeats its previous action ( $D$ in the former and $C$ in the latter). Conversely if it receives a low payoff $(P$ or $S)$ it prefers to change its behavior next time.

A general conclusion from these models is that strategies which support cooperation dominate in the long-run prospect.

This proposition is supported by several theoretical results. Fudenberg and Maskin (1990) and Binmore and Samuelson (1992) consider different variants of supergames with time-average payoffs, bounded rationality and symmetry of players and establish that the stable behavior is connected with the "utilitarian" payoff vectors which maximize the sum of player's payoffs. The notion of a utilitarian outcome generalizes the concept of cooperative behavior for symmetric two-player games. Thus, all mentioned papers make impression that evolution of behavior in repeated games leads to cooperation.

The main reason of such contrast to our conclusions is the bounded rationality of players in the mentioned models. The strategy combinations specified in the sections 3 , 4 become the dominance solutions only if behavior of the players is flexible with respect to the time till "the day of reckoning" $T$. If we consider players with bounded rationality, according to Kalai and Stanford (1988), then $m^{*}$ is not a dominance solution for sufficiently large $T$. But the specified flexibility seems to be typical for human behavior.

The reasonable question about the proposed scheme of behavior manipulation is if the active players can withstand it in the case where the imposed behavior is unprofitable for them, as in the example above. Of course, if some of them are sufficiently intelligent and have enough free money then they can create a "counter-manipulator" supporting cooperative behavior. One thing we would like to stress is that the cooperation (as well as other nice things) needs some regulation to support it.

\section{References}

Axelrod, R. (1984) "The evolution of Cooperation", New York: Basic Books.

Benoit, G.-P., Krishna, V. (1985) "Finitely Repeated Games", Econometrica, 53, 905-922

Binmore, K.J., Samuelson, L. (1992) "Evolutionary Stability in Repeated Games Played by Finite Automata", J. of Econ. Theory, 57, 278-305 
Fudenberg, D., Maskin, E. (1990) "Evolution and Cooperation in Noisy Repeated Games", New Developments in Economic Studies, 38, 1-12

Fudenberg, D., Maskin, E. (1986) "The Folk Theorem in Repeated Games with Discounting and with Incomplete Information", Econometrica, 54, 533-554

Moulin, H. (1984) "Dominance-solvability and Cournot-stability", Mathematical Social Sciences, 7, 83-102

Nachbar, J.H. (1990) "Evolutionary" Selection Dynamics in Games: Convergence and Limit Properties", Int. J. of Game Theory, 4, 25-55.

Nowak, M.A., Sigmund, K (1990) "The evolution in stochastic strategies in the prisoner's dilemma", Acta appl. math. 20, 247-265

Nowak, M.A., Sigmund, K (1992) "Tit for tat in heterogeneous populations.", Nature (London) 355: 250-253

Radner, R. (1980) "Collusive Behavior in Non-cooperative epsilon-equilibria of oligopolies with long but finite lives", J. of Econ. Theory, 22, 136-154.

Vasin, A.A. (1989) "Methods of Game Theory in Studies of Collective Behavioral Dynamics", Moscow University of Computational Mathematics and Cybernetics, Allerton press, New York, N 3, 51-55.

Vasin, A.A. (1990) "Evolutionary Model of Behavior in a Supergame", Moscow University Computational Mathematics and Cybernetics Bulletin, N 3, 54-60.

Vasin, A.A. (1994) "New Version of the Folk Theorem", 6th International Symposium on Dynamic Games and Applications, Preprint volume. Ed. by M. Breton and G. Zaccour, St-Jovite, Quebec, Canada

Vasin, A.A. (1997) "The Folk Theorem for Dominance Solutions", submitted to the IJGT. 